\title{
Epidemiologia e cuidado à Diabetes Mellitus praticado na Atenção Primária à Saúde: uma revisão narrativa
}

\author{
Xavéle Braatz Petermann ${ }^{1}$, Isadora Selistre Machado ${ }^{2}$, Bianca Nunes Pimentel ${ }^{3}$, \\ Silvana Basso Miolo ${ }^{4}$, Luciane Régio Martins ${ }^{5}$, Elenir Fedosse ${ }^{6}$
}

\begin{abstract}
RESUMO
Objetivo: Realizar uma busca de estudos epidemiológicos sobre Diabetes e sobre os cuidados prestados na Atenção Primária no Brasil. Metodologia: Revisão narrativa realizada a partir da busca dos termos "Atenção Primária à Saúde", "Atenção Básica à Saúde", "Epidemiologia" e "Estudos epidemiológicos" correlacionados com "Diabetes Mellitus", nas bases de dados SCIELO, LILACS e PUBMED. Resultados: Esta revisão revelou uma variação de $2 \%$ a $13 \%$ de Diabetes na população brasileira, nas três últimas décadas. Demonstrou maior prevalência em mulheres, em pessoas com sobrepeso/obesidade, em indivíduos sedentários, em sujeitos com menor nível de escolaridade e que 0 diagnóstico da doença é mais comum em idosos. Mostrou a importância das práticas educativas na Atenção Primária, com o objetivo de fortalecer 0 autocuidado. Considerações finais: A incidência e prevalência do Diabetes vêm aumentando no Brasil. Na Atenção Primária tal patologia deve ser acompanhada por uma equipe capacitada a desenvolver cuidados clínicos e práticas educativas.
\end{abstract}

Descritores: Diabetes Mellitus; Epidemiologia; Estudos Epidemiológicos; Atenção Primária à Saúde.

\section{Epidemiology and care in Diabetes Mellitus practiced in Primary Health Care: A narrative review}

\begin{abstract}
Object: Researching on epidemiologic studies about Diabetes Mellitus and about care in Primary Health Care in Brazil. Methodology: It is characterized as being a narrative review from researching terms such as "Primary Health Care", "Basic Health Care", "Epidemiology" and "Epidemiologic Studies" referred to "Diabetes Mellitus", in the scientific data bases SCIELO, LILACS and PUBMED. Results: This research revealed a variation between $2 \%$ and $13 \%$ of Diabetes in Brazilian population, in these last three decades. It showed larger prevalence in women, in people with overweight/obesity, in sedentary individuals, in people with low schooling level and also this sickness diagnosis is more common in aged population. It revealed the importance of the educative practices in Primary Health Care, which objective is to reinforce selfcare. Final considerations: Incidence and prevalence of Diabetes are increasing in Brazil. In Primary Health Care such pathology must be accompanied by a capable team for developing clinical care and educative practices.
\end{abstract}

Descriptors: Diabetes Mellitus; Epidemiology; Epidemiologic Studies; Primary Health Care.

\footnotetext{
${ }^{1}$ Graduanda em Fisioterapia na Universidade Federal de Santa Maria (UFSM), Santa Maria, RS, Brasil.

${ }^{2}$ Graduanda em Farmácia na Universidade Federal de Santa Maria (UFSM), Santa Maria, RS, Brasil.

${ }^{3}$ Graduada em Fonoaudiologia pela Universidade Federal de Santa Maria (UFSM), Santa Maria, RS, Brasil.

${ }^{4}$ Fisioterapeuta e Mestre em Engenharia de Produção pela Universidade Federal de Santa Maria (UFSM), Santa Maria, RS, Brasil.

${ }^{5}$ Graduada em Enfermagem na Universidade Federal de Santa Maria (UFSM), Santa Maria, RS, Brasil.

${ }^{6}$ Doutora em Linguística pela Universidade Estadual de Campinas (UNICAMP), Campinas, SP, Brasil.
} 


\section{Introdução}

As Doenças Crônicas Não-Transmissíveis (DCNT) como, por exemplo, Diabetes Mellitus (DM), doenças respiratórias e cânceres podem alterar de forma intensa a vida da maioria das pessoas ${ }^{1}$. A etiologia principal destas doenças não se encontra em fatores genéticos, e sim em vários fatores de riscos ambientais e comportamentais, como o tabagismo, a obesidade, a dislipidemia, a inatividade física e a alimentação inadequada, entre outros².

Tais doenças estão relacionadas às Atividades de Vida Diária (AVD) e, desde o estabelecimento do diagnóstico, podem produzir sentimentos de desespero e aflição, diminuindo a potência do indivíduo para agir e pensar. Por isso, essa situação impõe a necessidade de um cuidado integral à saúde ${ }^{1}$, o que implica uma nova atitude, ou seja, as pessoas com doenças crônicas deverão aprender a gerenciar suas vidas em um processo longitudinal que objetive qualidade e autonomia ${ }^{3}$.

As DCNT representaram, em 2007, cerca de $72 \%$ do total de mortes no Brasil ${ }^{4}$ e em 2003, o DM já representava 6\% do total de óbitos no País². Neste sentido, o DM encontra-se entre as principais causas de mortes, podendo alcançar 0 $7^{0}$ lugar dos óbitos em $2030^{5}$.

Estima-se que, mundialmente, o DM é uma doença que afeta 347 milhões, sendo que mais de $80 \%$ das mortes ocorrem em países de baixa e média renda. O DM possui alta incidência na população brasileira, revelando-se como um problema de grande importância social e para a saúde pública do País ${ }^{5}$.

Sabe-se que o DM é uma doença caracterizada pelo comprometimento do metabolismo da glicose, resultando em hiperglicemia crônica. Divide-se em Diabetes Tipo 1, Tipo 2, gestacional e outros tipos específicos. 0 DM Tipo 1 caracteriza-se pela destruição das células beta do pâncreas (geralmente causada por processo auto-imune), levando ao estágio de deficiência absoluta de insulina, sendo necessária a administração da insulina para prevenir cetoacidose, coma e até a morte. O DM tipo 2 caracteriza-se pela resistência à ação da insulina e a deficiência da insulina manifestase pela incapacidade de compensar essa resistência. Outros tipos de Diabetes são menos frequentes, e podem resultar de: defeitos genéticos da função das células beta e da ação da insulina, doenças no pâncreas exócrino, infecções, efeito colateral de medicamentos, etc ${ }^{3,6}$.

Entre os sintomas do DM estão a poliúria (excreção excessiva de urina), polidipsia (sede excessiva), fome constante, alterações na visão e fadiga; tais sintomas podem ocorrer repentinamente ${ }^{3}$.

Essa doença exige um acompanhamento regular e sistêmico por uma equipe multiprofissional de saúde que ofereça os recursos necessários para que a pessoa com DM possa manejar a patologia, ou seja, manter o autocuidado necessário para evitar o agravo da doença. Dessa maneira, a educação em saúde se constitui a base para o manejo e o domínio da enfermidade ${ }^{1}$.

Este estudo se propôs a realizar um levantamento de estudos epidemiológicos nacionais sobre Diabetes Mellitus e sobre os cuidados prestados aos sujeitos diabéticos na Atenção Primária à Saúde, no Brasil.

\section{Metodologia}

Esta pesquisa se caracteriza como uma revisão narrativa de artigos publicados nos últimos 10 anos (2003 a 2013) e indexados na base de dados científicas SCIELO, LILACS e PUBMED. Os termos utilizados para a busca dos artigos foram: "Atenção Primária à Saúde", "Atenção Básica à Saúde", "Epidemiologia" e "Estudos epidemiológicos" correlacionados com "Diabetes Mellitus". Também foi realizada uma busca nas referências dos artigos encontrados para suprir informações relacionadas ao tema. Os artigos selecionados retratam a incidência do DM nas últimas três décadas (década de 80, década de 90, 2003, 2006, 1998/2003/2008, 2008, 2010).

Foram encontrados 295 artigos nas bases de dados. Como critério de inclusão foi definido que os estudos deveriam tratar de investigações sobre a epidemiologia e sobre o cuidado à Diabetes praticado na atenção básica. Foram excluídos os artigos que tratavam da mera descrição da doença, bem como aqueles que abordavam o cuidado à DM em outros níveis de atenção à saúde (ambulatórios e hospitais) e, obviamente, os que apareceram repetidamente. Neste sentido, foram usados nesta revisão 31 estudos publicados no período de 2003 a 2013. 
Os resultados obtidos acompanhados das devidas discussões serão apresentados por meio de três categorias Incidência e prevalência do Diabetes Mellitus no Brasil, Comorbidades do Diabetes Mellitus e Cuidado no Diabetes Mellitus praticado na Atenção Primária à Saúde - visto ser estes os principais aspectos relativos ao tema desta revisão.

\section{Resultados e Discussões}

\section{Incidência e prevalência do Diabetes Mellitus no Brasil}

O DM possui etiologia multifatorial e se destaca entre as DCNT que representam um importante problema de Saúde Pública no mundo e no Brasil 7,8,9. Tais doenças decorrem, principalmente, da diminuição das taxas de nascimento e das de mortalidade associadas ao aumento da expectativa de vida. Esses eventos advêm da diminuição das taxas de mortalidade de crianças até cinco anos de idade, fato que aumenta a expectativa de vida de jovens e adultos, e da redução das doenças infecciosas. Esta mudança provoca uma transição epidemiológica e de carga de doenças, ou seja, uma mudança no perfil de saúde da população².

Os estudos epidemiológicos brasileiros apontam que nas últimas três décadas houve uma variação de $2 \%$ a $13 \%$ de pessoas com DM. Na década de 80 , o predomínio de Diabetes na população brasileira era de cerca de $2 \%{ }^{10}$; já na década de 90 do século passado encontrou-se um predomínio mais alto, variando entre $7 \%$ e 13\%10. Note-se que esses índices são muito superiores aos da Pesquisa de Saúde Mundial, realizada em 2003, que identificou um percentual de pessoas com Diabetes de 6,2\% ${ }^{11}$.

Um estudo ${ }^{12}$ baseado em inquérito de morbidade auto-referida, realizado em 2006, identificou uma prevalência de Diabetes em 5,3\%. A Pesquisa Nacional por Amostra de Domicílios (PNAD) ${ }^{13}$, realizada em 1998/2003/2008, sobre a prevalência do Diabetes no Brasil mostrou uma elevação de 2,9\% em 1998 para 4,3\% em 2008 (foram pesquisadas no ano de 1998 - 217.709, no ano de 2003 - 254.870 e no de 2008 - 271.677 pessoas com 18 anos ou mais de idade).

Estudos revelam como fatores de risco ao DM: a idade (predomínio acima de 60 anos) ${ }^{14,15,16}$, sobrepeso/obesidade ${ }^{14,16}$ e sedentarismo ${ }^{16}$. Nos estudos realizados a média de idade encontrada foi de 63,4 anos ${ }^{14}, 63,7$ anos ${ }^{15}$ e acima de 40 anos $^{16}$. Em relação ao sobrepeso e obesidade, o Índice de Massa Corporal (IMC) foi de 27,79 (sobrepeso) ${ }^{14}$. Em outro estudo, entre os diabéticos, $45,5 \%$ dos adolescentes, $36,0 \%$ dos adultos e $60,4 \%$ dos idosos apresentaram sobrepeso, enquanto $40,1 \%$ dos adultos eram obesos ${ }^{16}$. Pesquisa com dados secundários de 7.938 diabéticos (HiperDia) encontrou $42,9 \%$ de sedentários ${ }^{16}$.

Em todas as regiões brasileiras, em 2008, a prevalência de DM entre mulheres foi maior em comparação com os homens (a maior diferença entre os sexos foi na região Norte, a partir dos 60 anos). A região Sul apresentou prevalência mais elevada entre as mulheres de 70 a 79 anos, em torno de $21,5 \%$. No entanto, entre os homens da mesma faixa etária, a maior prevalência foi registrada na região Centro-Oeste, em torno de 17,3\%. Em ambos os sexos, o diagnóstico da doença se torna mais comum entre indivíduos com idade mais avançada, alcançando menos de 1,0\% dos indivíduos entre 18 e 29 anos e mais de 10,0\% dos indivíduos com 60 anos de idade ou mais ${ }^{13}$. Um estudo ${ }^{17}$, realizado em 2010, encontrou prevalência de $14,9 \%$ e 15,8\%, para homens e mulheres idosos, respectivamente.

Entre os estudos aqui analisados identificou-se que a prevalência de DM é maior na população feminina 8,14,15,16,18,19. Uma possível explicação para tais dados é o fato de as mulheres serem mais preocupadas com a saúde e, assim, procuram mais assistência e vivenciam maior autocuidado em relação ao homem.

Quanto à escolaridade, estudos ${ }^{14,15}$ revelam que quanto menor o nível de escolaridade e de informação maior é a tendência ao desenvolvimento da doença, logo evidenciou-se que a maioria dos casos de DM está entre analfabetos ou pessoas com baixo grau de escolaridade. A relevância de se obter conhecimento do nível de escolaridade das pessoas com DM está relacionada ao planejamento das estratégias de educação para 0 cuidado ${ }^{16}$.

\section{Comorbidades do Diabetes Mellitus}

Em estudos realizados no Brasil encontrou-se relação entre o DM e outras doenças decorrentes, agravantes ou agravadas por este: excesso de peso/obesidade ${ }^{20,21}$, glaucoma ${ }^{22}$, retinopatia diabética ${ }^{23,24}$, hipertensão arterial $^{13} \mathrm{e}$ sintomas depressivos ${ }^{25}$.

O sobrepeso ou a obesidade está presente em dois estudos sobre DM, tanto em relação aos fatores de riscos modificáveis, quanto ao tratamento através de uma abordagem educacional e alteração no estilo de vida. 
Um estudo ${ }^{20}$, com 399 pessoas idosas (227 idosas e 172 idosos), revelou que $73,6 \%$ das idosas e $66,9 \%$ dos idosos apresentavam sobrepeso ou eram obesos. Na amostra masculina, a prevalência de Diabetes era de $22,1 \%$, e entre as mulheres, de apenas 15,1\%. Este estudo encontrou associação significativa entre obesidade e a presença de DM, mas não revelou o percentual. Outro estudo indica que a prevalência da doença em idosos é $92 \%$ maior na presença do excesso de peso ${ }^{21}$.

O glaucoma é apontado na literatura como um fator de comorbidades e complicações relacionadas ao DM. Estudo com 50 pessoas com DM tipo 2 não houve diferença significativa entre a presença de glaucoma e DM (apenas 10\% da amostra apresentou glaucoma), ao comparar com a prevalência dessa patologia na população geral. Entretanto, traz em sua discussão uma prevalência de glaucoma $40 \%$ maior nos diabéticos ${ }^{22}$.

O DM também pode causar complicações microvasculares. A mais comum é a retinopatia diabética (RD), que ocorre tardiamente - após 20 anos de doença em mais de 90\% das pessoas com DM tipo 1 e em 60\% dos de tipo 2 . Essa complicação está presente, sobretudo em pacientes com longo tempo de doença e difícil controle glicêmico. A RD é a causa mais frequente de cegueira adquirida que compromete pessoas em idade produtiva, constituindo importante fator de morbidade com elevado impacto social econômico ${ }^{23}$.

Uma pesquisa ${ }^{24}$, com 120 sujeitos com DM tipo 2, identificou prevalência de RD em $38,4 \%$ da população pesquisada. Quanto ao sexo a RD foi encontrada em $43 \%$ dos homens e 33,8\% das mulheres. Quanto ao tipo de RD, 34,2\% apresentaram RD não Proliferativa (classificada em leve, moderada e intensa dependendo da presença de microaneurismas, hemorragias intrarretinianas, veias em rosário e/ou anormalidades microvasculares intrarretinianas) e apenas 4,2\% indicaram RD Proliferativa (crescimento de vasos anormais em resposta a isquemia de vasos da retina). Convém ressaltar que os atendimentos relacionados com DM pelo SUS cresceram consideravelmente nos últimos anos, de aproximadamente 190 mil no ano de 1998 atingindo 5,86 milhões de atendimentos em $2004^{24}$.

A já referida pesquisa - PNAD - realizada em 1998/2003/2008 ocupou-se, entre outros fatos, da evolução do DM associado à hipertensão arterial no Brasil, e encontrou que o coeficiente de prevalência padronizado de diabetes associado à hipertensão, elevou-se de 1,7\% em 1998 para 2,8\% em 2008 ${ }^{13}$.

Um estudo ${ }^{25}$ realizado com 145 pessoas com DM tipo 2 buscou averiguar a associação entre sintomas depressivos e variáveis sociodemográficas e clínicas, bem como a adesão ao tratamento medicamentoso. Os resultados demonstraram que $33,1 \%$ dos pesquisados apresentaram sintomas depressivos e que quanto maior era a medida da depressão, menor se apresentava a adesão ao tratamento. $\mathrm{Na}$ avaliação, as pessoas que não praticavam atividade física semanalmente apresentaram maior tendência a possuir os sintomas depressivos ${ }^{25}$. Neste sentido, deve-se refletir sobre a importância destas ações na atenção básica, com o intuito de diminuir o percentual de sintomas depressivos em indivíduos com Diabetes.

\section{Cuidado do Diabetes Mellitus praticado na Atenção Primária à Saúde}

O manejo do DM deve ser feito dentro de um sistema de saúde organizado em rede, em que a equipe deve atuar de maneira integrada, tendo como alicerce do cuidado o nível primário de atenção à saúde (a "porta de entrada" para 0 sistema de saúde) $)^{3}$.

Na Atenção Primária à Saúde (APS) são utilizadas intervenções com enfoque individual e coletivo ${ }^{26}$, visando atender os problemas mais comuns da população, entre eles, as DCNT, as quais por envolverem causas múltiplas, requerem uma atenção integral ${ }^{3,27}$.

Há um estudo ${ }^{28}$ que refere as oficinas educativas em DM, realizadas pelos profissionais de saúde, como importante dispositivo para o cuidado em saúde. 0 referido estudo revela a preocupação dos profissionais com relação à importância da integração da equipe para a promoção e educação em DM. Explica, ainda, que as oficinas focalizam o autocuidado relacionado à doença, valorizando a condição de a pessoa compreender e avaliar a sua condição. Desta forma, proporciona-se apoio integral (apoio emocional, clínico, ampliação de conhecimentos e habilidades) para que a pessoa alcance os objetivos - descobrir e desenvolver a autonomia para controlar a sua doença ${ }^{28}$.

Outro estudo ${ }^{29}$, na perspectiva do cuidado empoderador, pauta pela participação ativa e interação, através da parceria entre o usuário, profissional e família, aumentando a probabilidade no alcance do sucesso nas ações desenvolvidas no DM. Esclarece que as ações desenvolvidas devem estimular a confiança mútua e a co-responsabilidade (na parceria as pessoas envolvidas são beneficiadas pelas trocas de conhecimento e de experiência de vida ${ }^{29}$. 
Constatou-se que, ressaltando o cuidado co-responsável e a produção de autonomia, o novo modelo de saúde para condições crônicas abrange uma estrutura que deve incluir uma aliança entre usuários, familiares, equipes de saúde, instituições e comunidade, auxiliando na prevenção e contensão de agravos das doenças crônicas ${ }^{30}$.

A utilização de práticas educativas - realizadas por uma equipe de saúde - tem por intuito melhorar o conhecimento das pessoas sobre o DM, assim como incentivá-las a ter hábitos de vida saudáveis (fatos que melhoram a sua qualidade de vida, aumentando a sua autonomia perante a patologia) ${ }^{29,31}$.

No sentido acima, são indispensáveis os programas voltados para educação em DM com a finalidade de evitar possíveis complicações, pois o DM ainda representa uma patologia de grande morbimortalidade no mundo e no Brasil, existindo sempre a necessidade de novos estudos que contemplem ações que possibilitam melhorar a qualidade de vida dos indivíduos com DM, retardando o aparecimento das complicações, principalmente as crônicas ${ }^{8}$.

O modelo de saúde, vigente no Brasil, organizado em três níveis de atenção (atenção básica, secundária e terciária), consegue, através das ações em APS, um maior monitoramento do DM, pois a equipe de saúde pode, em parceria com grupos comunitários e familiares, auxiliar as pessoas na busca de soluções, diminuindo os seus problemas de saúde. Além disso, contemplando a integralidade da assistência, os profissionais de saúde podem atuar em todas as fases do processo saúde-doença ${ }^{30}$.

Estudo $^{30}$ realizado na Saúde da Família em Ribeirão Preto - SP refere que além da atenção aos aspectos técnicos do Diabetes - manutenção dos níveis glicêmicos e do monitoramento das complicações decorrentes da doença - as atividades realizadas pela equipe de saúde na APS devem também considerar os aspectos emocionais decorrentes da patologia. Torna-se importante a compreensão da repercussão de uma doença crônica para o próprio sujeito e para a sua família, para que assim seja planejado um plano de tratamento para o controle do Diabetes e para a prevenção das suas complicações. O plano de tratamento também deve integrar os familiares, considerados importantes para a adesão do indivíduo ao tratamento. Somente de maneira integrada e articulada é que se consegue refletir sobre a controle adequado e, como consequência, a melhor qualidade de vida para pessoas com $\mathrm{DM}^{30}$.

\section{Considerações Finais}

A revisão de literatura pertinente ao DM revelou que tal doença representa um importante problema de Saúde Pública no mundo e, especialmente, no Brasil, à medida que sua incidência, prevalência e comorbidades na população brasileira vêm aumentando ao longo dos anos.

As pesquisas demonstraram uma variação de $2 \%$ a $13 \%$ de pessoas com DM nas últimas três décadas no Brasil, mostraram a prevalência da doença em mulheres, idosos, pessoas com sobrepeso/obesidade, indivíduos sedentários, menor nível de escolaridade e revelaram que as principais comorbidades do Diabetes são excesso de peso/obesidade, glaucoma, retinopatia diabética, hipertensão arterial e sintomas depressivos.

Os estudos epidemiológicos são importantes para fortalecer o cuidado em saúde de pessoas com DM, pois identificam os casos e os fatores de risco para a doença.

ODM tem naAtenção Primária espaço privilegiado para o acompanhamento de tal patologia que deve ser acompanhada por uma equipe capacitada a desenvolver cuidados clínicos e práticas educativas voltadas à Promoção da Saúde que implica a prevenção do DM (atuando sobre os fatores de riscos modificáveis) e a prevenção de agravos decorrentes dele.

A revisão aqui realizada permitiu identificar a importância de que o cuidado prestado a pessoas com DM deve ser conduzido por uma equipe multiprofissional atuando interdisciplinarmente para poder responder às características individuais, tanto do perfil da comunidade, como do perfil da própria equipe de saúde. Estes fatores são indispensáveis para aumentar a resolubilidade na rede básica (na APS), ou seja, redução do número de casos novos de DM, bem como do número de encaminhamentos para outros níveis de atenção em saúde decorrentes do aumento do grau de coresponsabilização entre as equipes de saúde e os usuários dos serviços de APS. 


\section{Referências Bibliográficas}

1. Oliveira KCS, Zanetti ML. Conhecimento e atitude de usuários com diabetes mellitus em um Serviço de Atenção Básica à Saúde. Rev Esc Enferm USP. 2011; 45(4): 862-8.

2. Banco Mundial. Unidade de Gerenciamento do Brasil. Unidade de Gestão do Setor de Desenvolvimento Humano. Região da América Latina e do Caribe. Enfrentando o Desafio das Doenças Não Transmissíveis no Brasil. Documento do Banco Mundial. Relatório № 32576; 2005. [acesso 2013 abr 25]. Disponível em: ftp://ftp.cve.saude.sp.gov.br/doc_tec/ cronicas/relatorio_bancomundial.pdf

3. Ministério da Saúde. Secretaria de Atenção à Saúde (BR). Departamento de Atenção Básica. Diabetes Mellitus / Ministério da Saúde, Secretaria de Atenção à Saúde, Departamento de Atenção Básica. - Brasília (DF): Ministério da Saúde; 2006. 56 p. 4. Schmidt MI, Duncan BBE, Silva GA, Menezes AM, Monteiro CA, Barreto SM, Et al. Chronic noncommunicable diseases in Brazil: burden and current challenges. Lancet. 2011; 377(9781): 1949-61.

5. World Health Organization. Diabetes: diabetes facts. Fact sheet 2011 Jan № 312.

6. Schmidt MI. Diabetes Melito: diagnóstico, classificação e abordagem inicial. In: Duncan BB; Schmidt Ml; Giugliani ERJ. Medicina ambulatorial: condutas de atenção primária baseadas em evidências. 3a ed. Porto Alegre (RS): Artmed; 2004.p.669-76.

7. Dias JCR, Campos JADB. Diabetes mellitus: razão de prevalências nas diferentes regiões geográficas no Brasil, 2002 - 2007. Ciênc. saúde coletiva. 2012; 17(1): 239-244.

8. Morais GFC, Soares MJGO, Costa MML, Santos IBC. O diabético diante do tratamento, fatores de risco e complicações crônicas. Rev. enferm. UERJ. 2009; 17(2): 240-5.

9. Santos L, Torres HC. Práticas educativas em Diabetes Mellitus: compreendendo as competências dos profissionais da saúde. Texto Contexto Enferm. 2012; 21(3): 574-80.

10. Westphal MF, Mendes R, Lima CWV, Martins CA, Ferdinando DC. Informações básicas sobre as doenças não transmissíveis e fatores de risco para elaboração de análises econômicas - incluindo prevalência, duração das doenças, mortalidade e outras informações básicas. Trabalho de base do Banco Mundial. São Paulo; Faculdade de Saúde Pública; 2004. 111p.

11. Organização Panamericana de Saúde. Doenças crônicas degenerativas e obesidade: estratégia mundial sobre alimentação, atividade física e saúde. Brasília: OPAS; 2003.

12. Schmidt, MI. Prevalência de diabetes e hipertensão no Brasil baseada em inquérito de morbidade auto-referida, Brasil, 2006. Rev Saúde Pública. 2009; 43(Supl 2):74-82.

13. Freitas LRS, Garcia LP. Evolução da prevalência do diabetes e deste associado à hipertensão arterial no Brasil: análise da Pesquisa Nacional por Amostra de Domicílios, 1998, 2003 e 2008. Epidemiol. Serv. Saúde, 2012; 21(1):7-19. 14. Sampaio FAA, Melo RP, Rolim ILTP, Siqueira RC, Ximenes LB, Lopes MVO. Avaliação do comportamento de promoção da saúde em portadores de Diabetes Mellitus. Acta Paul Enferm. 2008; 21(1): 84-8.

15. Torres HC, Roque C, Nunes C. Visita domiciliar: Estratégia educativa para o autocuidado de clientes diabéticos na atenção básica. Rev. enferm. UERJ. 2011; 19(1): 89-93.

16. Ferreira CLRA, Ferreira MG. Características epidemiológicas de pacientes diabéticos da rede pública de saúde análise a partir do sistema HiperDia. Arq Bras Endocrinol Metab. 2009; 53(1): 80-6.

17. Francisco PMSB, Belon AP, Barros MBA, Carandina L, Alves MCGP, Goldbaum M, Et al. Diabetes auto-referido em idosos: prevalência, fatores associados e práticas de controle. Cad. Saúde Pública. 2010; 26(1):175-184.

18. Mielczarski RG, Costa JSD, Olinto MTA. Epidemiologia e organização de serviços de saúde: diabetes mellitus numa comunidade de Porto Alegre. Ciênc. saúde coletiva. 2012; 17(1): 71-78.

19. Cotta RMM, Reis RS, Batista KCS, Dias G, Alfenas RCG, Castro FAF. Hábitos e práticas alimentares de hipertensos e diabéticos: repensando o cuidado a partir da atenção primária. Rev. Nutr. 2009; 22(6): 823-835.

20. Aurichio TR, Rebelatto JR, Castro AP. Obesidade em idosos do Município de São Carlos, SP e sua associação com diabetes melito e dor articular. Fisioter Pesq. 2010; 17(2): 114-7.

21. Pelegrini A, Coqueiro RA, Petroski EL, Benedetti TRB. Diabetes Mellitus auto-referido e sua associação com excesso de peso em idosos. Rev Bras Cineantropom Desempenho Hum. 2011; 13(6):442-447.

22. Coblentz J, Pires MLE, Oliveira D, Lebeis K, Terrezo L. Prevalência de diabetes mellitus tipo 2 e outros fatores de risco associados em pacientes com glaucoma. Rev Bras Oftalmol. 2010; 69 (1): 33-5.

23. Sociedade Brasileira de Diabetes. Diabetes na prática clínica, 2011. [acesso 2013 Jun 01]. Disponível em: http:// www.diabetesebook.org.br/. 
24. Jos BS. Prevalência de retinopatia diabética na população portadora de diabetes mellitus tipo 2 do município de Luzerna - SC. Arq Bras Oftalmol. 2010; 73(3): 259-65.

25. Braz JM, Silva MR, Gois CFL, Braz TM, Santos V, Silva LASM. Sintomas depressivos e adesão ao tratamento entre pessoas com diabetes mellitus tipo 2. Rev Rene. 2012; 13(5):1092-9.

26. Brasil. Ministério da Saúde. Portaria n. 2.488, de 21 de outubro de 2011. Aprova a Política Nacional de Atenção Básica estabelecendo a revisão de diretrizes e normas para a organização da Atenção Básica, para a Estratégia Saúde da Família (ESF) e o Programa de Agentes Comunitários de Saúde (PACS). Diário Oficial da União, Brasília, 21 out. 2011. Seção 1, n² 204, p. 1-37.

27. Guidoni CM, Olivera CMX, Freitas O, Pereira LRL. Assistência ao diabetes no Sistema Único de Saúde: análise do modelo atual. Braz. J. Pharm. Sci. 2009; 45(1): 37-48.

28. Torres HC, Amaral MA, Amorim MM, Cyrino AP, Bodstein R. Capacitação de profissionais da atenção primária à saúde para educação em Diabetes Mellitus. Acta Paul Enferm. 2010; 23(6): 751-6.

29. Hammerschmidt KSA, Lenardt MA. Tecnologia educacional inovadora para o empoderamento junto a idosos com Diabetes Mellitus. Texto Contexto Enferm. 2010; 19 (2): 358-65.

30. Silva LMC, Palha PF, Barbosa GR, Protti ST, Ramos AS. Aposentados com diabetes tipo 2 na Saúde da Família em Ribeirão Preto, São Paulo - Brasil. Rev Esc Enferm USP. 2010; 44 (2): 462-8.

31. Grillo MFF, Neumann CR, Scain SF, Rozeno RF, Gross JL, Leitão CB. Efeito de diferentes modalidades de educação para 0 autocuidado a pacientes com diabetes. Rev assoc med bras. 2013; 59(4):400-5.

\section{Xavéle Braatz Petermann}

Endereço para correspondência - Avenida Roraima, n 1000, apto 4330, Bairro: Camobi, CEP: 97105-900, Cidade: Santa Maria, RS, Brasil.

E-mail: xavelepetermann@gmail.com

Lattes: http://lattes.cnpq.br/1155618369131325

Isadora Selistre Machado - isadoraselistre@gmail.com

Bianca Nunes Pimentel - pimentelbnc@hotmail.com

Silvana Basso Miolo - sbmiolo@hotmail.com

Luciane Régio Martins - luhumaniza2009@gmail.com

Elenir Fedosse - efedosse@gmail.com

Enviado em 20 de julho de 2014. Aceito em 07 de abril de 2015. 
